\title{
Routine versus ad hoc screening for acute stress following injury: who would benefit and what are the opportunities for prevention
}

\author{
Nathaniel Bell ${ }^{1,4}$, Boris Sobolev², Stephen Anderson ${ }^{3}$, Robert Hewko ${ }^{3}$ and Richard K Simons ${ }^{1 *}$
}

\begin{abstract}
Background: Screening for acute stress is not part of routine trauma care owing in part to high variability of acute stress symptoms in identifying later onset of posttraumatic stress disorder (PTSD). The objective of this pilot study was to assess the sensitivity, specificity, and power to predict onset of PTSD symptoms at 1 and 4 months using a routine screening program in comparison to current ad hoc referral practice.

Methods: Prospective cross-sectional observational study of a sample of hospitalized trauma patients over a four-month period from a level-I hospital in Canada. Baseline assessments of acute stress (ASD) and subsyndromal ASD (SASD) were measured using the Stanford Acute Stress Reaction Questionnaire (SASRQ). In-hospital psychiatric consultations were identified from patient discharge summaries. PTSD symptoms were measured using the PTSD Checklist-Specific (PCL-S). Post-discharge health status and health services utilization surveys were also collected.

Results: Routine screening using the ASD (0.43) and SASD (0.64) diagnoses were more sensitive to PTSD symptoms at one month in comparison to ad hoc referral $(0.14)$ and also at four months $(0.17,0.33$ versus 0.17$)$. Ad hoc referral had greater positive predictive power in identifying PTSD caseness at 1 month (0.50) in comparison to the ASD (0.46) and SASD (0.43) diagnoses and also at 4 months (0.67 versus 0.25 and 0.29 ).

Conclusions: Ad hoc psychiatric referral process for acute stress is a more conservative approach than employing routine screening for identifying persons who are at risk of psychological morbidity following injury. Despite known limitations of available measures, routine patient screening would increase identification of trauma survivors at risk of mental health sequelae and better position trauma centers to respond to the circumstances that affect mental health during recovery.
\end{abstract}

Keywords: Trauma systems, Traumatic stress/PTSD, Screening

\section{Background}

The potential to identify trauma survivors at risk for developing PTSD and thereby enable short- and long-term mental health intervention early on was a core driving mechanism in introducing acute stress disorder (ASD) into the Diagnostic and Statistical Manual of Mental Disorders (DSM-IV) taxonomy in 1994 [1]. An estimated ten to forty percent of civilian injury survivors go on to display symptoms consistent with PTSD, [2-6] of which the probability of remission has been estimated at $18 \%$ to

\footnotetext{
*Correspondence: richard.simons@vch.ca

'Department of Surgery, University of British Columbia, 855 West 10th

Avenue, Vancouver, British Columbia V5Z 1 M9, Canada

Full list of author information is available at the end of the article
}

$38 \%[7,8]$. However, the ASD diagnosis has been criticized owing to marked variability in predicting later onset of PTSD and was not recommended to be retained in the DSM-V as a predictor of subsequent PTSD [9]. While changes to the diagnosis have been made in the recent release of the DSM-V, there remains little evidence that the ASD diagnosis is accurately predicting longer-term PTSD.

These critiques have implications for how clinicians identify and manage psychological health after injury. In Canada, early screening for acute stress is not part of routine trauma care. Although it is well established that early identification of individuals at risk for PTSD is important for minimizing psychological morbidity, $[10,11]$ trauma centers currently rely on ad hoc referral practices 
for identifying those individuals who display symptoms of acute stress. It is not clear whether current practices are an appropriate substitute to administering what are essentially very low-cost and easily administered self-reported surveys of acute stress. In addition, there has been a lack of literature evaluating the clinical utility of routine screening for identifying ongoing life stressors that may impact mental health. This is a significant limitation because persons with mental health illnesses often do not receive treatment, [12] are less likely to see health care specialists [13] and experience emotional disabilities often in parallel with substantial physical, social, and economic disadvantages $[14,15]$.

We conducted a prospective observational pilot study with adult injury survivors treated at Vancouver General Hospital, British Columbia, Canada to assess whether routine screening for acute stress improved identification of patients who later went on to display PTSD symptoms compared with identification using ad hoc psychiatric referral practices currently in place. These evaluations were contextualized against patient self-reported experiences and expectations for recovery in effort to outline future surveillance and intervention opportunities for minimizing psychiatric morbidity after injury.

\section{Methods}

This prospective cross-sectional observational pilot study targeted all residents of British Columbia and the Yukon ages 19 and older who were referred or admitted directly to Trauma Services, Vancouver General Hospital following injury. Patients were recruited over a consecutive enrollment period between June and November, 2011. Excluded were persons who had a hospital stay $<24$ hours and those with a Glasgow Coma Scale $<15$ at discharge. All patients actively consented to participate while in hospital. Interviews took place on the ward and on the day prior to discharge whenever possible to ensure minimal impact on patient care. Follow-up interviews were conducted 1 and 4 months after the initial interview. After each interview participants were asked if they consented to further follow-up. The follow-up period of 1 and 4 months was selected based on recommendations from the literature [16]. This study was registered and approved by the UBC Behavioral Research Ethics Board and the Vancouver Coastal Health Research Institute, which represents Vancouver General Hospital.

\section{Acute stress survey}

Each patient completed a self-reported assessment of acute stress using the Stanford Acute Stress Reaction Questionnaire (SASRQ) [17]. The SASRQ is a 30-item closed-ended self-report questionnaire used to assess symptoms of ASD following a traumatic event $[5,18,19]$. The SASRQ uses DSM-IV criteria for diagnosing ASD.
The SASRQ includes measures of acute dissociation (Cluster B; 10 items), re-experiencing (Cluster C; 6 items), avoidance (Cluster D; 6 items), anxiety and hyper-arousal (Cluster E; 6 items), and impairment in functioning (Cluster F; 2 items). Respondents were asked to report the frequency with which they experienced each item using a five-point Likert scale, spanning from: (0) have/did not experience, (1) very rarely experienced, (2) rarely experienced, (3) sometimes experienced, (4) often experienced, and (5) very often experienced. The diagnostic cutoff for ASD requires each person to assign a value of 3 or higher to at least three of the ten dissociative criteria, and a score of three or higher for at least one of symptom clusters $C, D, E$, and F. The criteria for SASD only requires meeting the diagnostic cut-off for symptom clusters $\mathrm{C}, \mathrm{D}$, and E. Positive SASD events were derived from the original SASRQ response score scores.

\section{PTSD survey}

PTSD symptoms at one and four months were identified using the PCL-S (Specific) [20]. The PCL-S is a selfreport measure developed by the US National Center for PTSD containing the 17 DSM-IV symptoms of PTSD. At each interview, participants were asked how often they have been bothered by each symptom in the past month. Responses were provided using a 5-point Likert scale, spanning from: (1) not at all, (2) a little bit, (3) moderately, (4) quite a bit, or (5) extremely. The PCL-S is scored by using a summation of the individual 17 symptoms. A cut-off score of 44 was used to identify positive PTSD symptoms. This score was chosen based on a previous sensitivity analysis using Clinician Administered PTSD Scale (CAPS) scores with 40 motor vehicle and sexual assault survivors [21].

\section{Behavior, service utilization, and socio-economic survey} During the baseline interview participants were asked to respond to their current employment status, level of educational attainment, living arrangement, housing tenure and if they had sought treatment for anxiety or depression in the past year. At follow-up, participants were asked if they had a post-discharge consultation with a health care provider about their health as a result of their injury, whether they were taking medication for stress, or had consulted a medical professional about anxiety or depression as a result of their injury. At the conclusion of each interview participants were asked whether they felt as emotionally or physically healthy as they were before injury, and if not, whether these experiences had been expected. All participants were asked to openly comment on these experiences as well as any barriers that they had experienced in attempting to access health care services. 


\section{Statistical analysis}

The principle outcome measures for this study were a baseline diagnosis of ASD and SASD using routine and ad hoc screening, as well the sensitivity and specificity of these practices in predicting PTSD symptomatology at 1 and 4 months. Psychiatric referrals and consultations were identified from reviewing hospital discharge summaries. Differences between means of continuous variables were examined using a 2-tailed independent samples $t$ test, and differences in proportions of categorical variables were examined using a $\chi^{2}$ test. To account for potential type-I errors in the subgroup analysis all goodness of fit tests were reported using a continuity-adjusted $\chi^{2}$. All statistical analyses were generated using SAS software, Version 9.2 for Windows [22].

\section{Results}

A total of 91 of 120 eligible patients we approached consented to participate in this pilot study, for an overall opt-in consent rate of $76 \%$. The study participation rate at one month was $61 \%(n=56)$ and $47 \%(n=43)$ after four months. Six participants were lost to follow-up at month 1, but returned a survey at month 4 . These response scores were included in the analyses as there was no socio-demographic, health status, or injury-related differences between those who partially or fully participated in follow-up. Socio-demographic, health status, and injury-related characteristics of all eligible patients categorized by consent, participation, and loss to follow-up status are shown in Table 1.
A total of 34 of the 91 participants (37\%) scored positive for SASD and 22 participants (24\%) scored positive for ASD using the SASRQ questionnaire during the baseline (in-hospital) assessment. Median hospital length of stay prior to completing the SASRQ was 7 days. All participants were screened for ASD within the time range required for an ASD diagnosis $(2-31)$ days. A total of 8 of the 91 participants (9\%) received a referral for a psychiatric consultation prior to discharge from Trauma Services. All eight participants had positive SASD scores and five reported positive ASD scores.

At one month, 14 of the 56 participants (25\%) scored positive for PTSD symptoms based on the PCL-S assessment. At four months 12 of the 43 participants (28\%) submitted positive PTSD assessment scores. Five of the 14 participants (36\%) who reported positive PTSD scores at 1 month were lost to follow-up.

To assess the optimal predictive value of the ASD diagnosis we conducted a sensitivity, specificity, and power to predict test using individual ASD symptoms and different groupings for each symptom cluster. Tables 2 and 3 show the sensitivity, specificity, and power to predict PTSD symptoms at 1 and 4 months using various diagnostic groupings. Overall, employing different symptom groupings did not substantially improve either the sensitivity or predictive power of the ASD diagnosis. At month 1, the individual symptom clusters were more sensitive predictors than either the ASD or subsyndromal ASD diagnosis, but had lower predictive power. In contrast, at month 4 the individual symptom clusters were more sensitive

Table 1 Characteristics of the 120 patients who were eligible for enrollment by their consent to participate and by retention

\begin{tabular}{|c|c|c|c|c|c|c|}
\hline Variable & $\begin{array}{c}\text { Consenters } \\
(n=91)\end{array}$ & $\begin{array}{l}\text { Non-consenters } \\
(n=29)\end{array}$ & $p$ value & $\begin{array}{l}\text { Partial/full participation } \\
(n=63)\end{array}$ & $\begin{array}{l}\text { Lost to follow-up } \\
(n=28)\end{array}$ & $\overline{p \text { value }}$ \\
\hline \multicolumn{7}{|l|}{ Socio-demographics } \\
\hline Mean (SD) age (years) & $45(19)$ & $37(16)$ & 0.05 & $45(18)$ & $45(19)$ & 0.87 \\
\hline Male & $63(69)$ & $21(84)$ & 0.23 & $45(72)$ & $18(62)$ & 0.44 \\
\hline Having no high school diploma & $11(12)$ & - & - & $6(10)$ & $5(18)$ & 0.45 \\
\hline Unememployed at time of injury & $15(16)$ & - & - & $9(14)$ & $6(21)$ & 0.66 \\
\hline Currently living alone & $27(30)$ & - & - & $13(21)$ & $14(48)$ & 0.02 \\
\hline Living in rented housing & $61(67)$ & - & - & $39(63)$ & $22(76)$ & 0.32 \\
\hline \multicolumn{7}{|l|}{ Health status } \\
\hline Treated for anxiety or depression in the last year & $17(19)$ & - & - & $12(20)$ & $5(17)$ & 1.00 \\
\hline Scored positive for ASD & $22(24)$ & - & - & $13(21)$ & $9(31)$ & 0.43 \\
\hline Scored positive for SASD & $34(37)$ & - & - & $21(34)$ & $13(45)$ & 0.44 \\
\hline \multicolumn{7}{|l|}{ Injury-related } \\
\hline Positive blood alcohol (BAC) result & $13(16)$ & $1(29)$ & 0.28 & $8(15)$ & $5(20)$ & 0.80 \\
\hline Positive toxicology result & $9(11)$ & $9(37)$ & 0.01 & $6(11)$ & $3(12)$ & 1.00 \\
\hline Mean (SD) injury severity score (ISS) & $22(13)$ & $22(10)$ & 0.98 & $23(13)$ & $20(12)$ & 0.33 \\
\hline Intentional injury & $14(17)$ & $4(17)$ & 1.00 & $4(7)$ & $10(37)$ & $<0.001$ \\
\hline
\end{tabular}

Values reported are counts (\% of group total) unless otherwise stated. 
Table 2 Sensitivity, specificity, and power to predict 9 PTSD cases at month one using the ASD symptomatology

\begin{tabular}{|c|c|c|c|c|}
\hline DSM-IV ASD criteria & Sensitivity & Specificity & PPV & NPV \\
\hline \multicolumn{5}{|l|}{$\begin{array}{l}\text { ASD symptoms using routine } \\
\text { screening }\end{array}$} \\
\hline B. Dissociation & 0.78 & 0.43 & 0.31 & 0.86 \\
\hline C. Re-experiencing/Intrusion & 0.78 & 0.67 & 0.44 & 0.90 \\
\hline D. Avoidance & 0.78 & 0.48 & 0.33 & 0.87 \\
\hline E. Anxiety/Hyperarousal & 0.93 & 0.19 & 0.28 & 0.89 \\
\hline F. Impairment & 0.86 & 0.17 & 0.26 & 0.78 \\
\hline \multicolumn{5}{|l|}{$\begin{array}{l}\text { Subsyndromal ASD using } \\
\text { routine screening }\end{array}$} \\
\hline$C+D+E$ & 0.64 & 0.71 & 0.43 & 0.86 \\
\hline \multicolumn{5}{|l|}{$\begin{array}{l}\text { ASD diagnosis using routine } \\
\text { screening }\end{array}$} \\
\hline $\mathrm{B}+\mathrm{C}+\mathrm{D}+\mathrm{E}+\mathrm{F}+2$ day & 0.43 & 0.83 & 0.46 & 0.81 \\
\hline \multicolumn{5}{|l|}{ Ad hoc referral practice } \\
\hline Consultation with psychiatry & 0.14 & 0.95 & 0.50 & 0.77 \\
\hline
\end{tabular}

PPV: positive predictive value; NPV: negative predictive value. The accuracy of PTSD classification characterized by ad hoc referral with psychiatry was measured in reference to the participants SASRQ response score.

predictors than either the ASD or subsyndromal ASD diagnosis and resulted in stronger predictive power, on average, in identifying participants who scored positive for PTSD symptoms. In both time periods, ad hoc referral practices were less sensitive than routine screening for identifying individuals who went on to display PTSD symptoms, but resulted in greater positive predictive power for identifying persons who exhibited PTSD symptoms.

Table 3 Sensitivity, specificity, and power to predict 10 PTSD cases at month four using the ASD symptomatology

\begin{tabular}{lcccc}
\hline DSM-IV ASD criteria & Sensitivity & Specificity & PPV & NPV \\
\hline $\begin{array}{l}\text { ASD symptoms using routine } \\
\text { screening }\end{array}$ & & & & \\
$\quad$ B. Dissociation & 0.75 & 0.39 & 0.32 & 0.80 \\
$\quad$ C. Re-experiencing/Intrusion & 0.50 & 0.58 & 0.32 & 0.75 \\
$\quad$ D. Avoidance & 0.58 & 0.55 & 0.33 & 0.77 \\
$\quad$ E. Anxiety/Hyperarousal & 0.92 & 0.13 & 0.29 & 0.80 \\
$\quad$ F. Impairment & 0.83 & 0.13 & 0.27 & 0.67 \\
$\begin{array}{l}\text { Subsyndromal ASD using } \\
\text { routine screening }\end{array}$ & & & & \\
$\quad$ C + D + E & 0.33 & 0.68 & 0.29 & 0.72 \\
$\begin{array}{l}\text { ASD diagnosis using routine } \\
\text { screening }\end{array}$ & & & & \\
$\quad$ B + C + D + E + F + 2 day & 0.17 & 0.81 & 0.25 & 0.71 \\
Ad hoc referral practice & & & & \\
$\quad$ Consultation with psychiatry & 0.17 & 0.97 & 0.67 & 0.75
\end{tabular}

PPV: positive predictive value; NPV: negative predictive value. The accuracy of PTSD classification characterized by ad hoc referral with psychiatry was measured in reference to the participants SASRQ response score.
Characteristics of participants by response scores to the PCL-S self-assessment test at month 1 and month 4 are shown in Table 4. At month 1, persons who scored positive for PTSD could be differentiated from respondents who were symptom negative when contrasted by gender (43\% male vs $78 \%$ male, $p 0.03)$ and by injury severity (ISS 15 vs ISS $24 p$ 0.03). No differences in health behavior, expectations, or health service utilization were observed. By month four, persons who scored positive for PTSD symptoms experienced more barriers accessing care ( $42 \%$ vs $10 \%, p$ 0.05), having unexpected physical and emotional pain ( $91 \%$ vs $42 \% ; p 0.02$ ), and displayed greater medication use for stress or anxiety ( $42 \%$ vs $6 \%, p 0.02)$.

There was an indication that participants who scored positive for PTSD symptoms at month 4 were primarily self-coping as only half of the patient population had reported discussing concerns about stress with their health care provider. When asked why, participants reported that they did not know with whom to talk with about their stress or how to approach their physician about problems they were experiencing. Differences were also observed with regards to satisfaction with current level of social support, with $33 \%$ of persons who displayed PTSD symptoms reporting they did not have adequate social support compared to $7 \%$ of persons without PTSD symptoms $(p 0.04)$. When asked to comment on barriers experienced accessing care, participants listed a lack of extended health insurance and lack of income as the leading cause contributing to failure to see or consult with a health care provider about stress or anxiety.

\section{Discussion}

Both routine screening and ad hoc referral for acute stress resulted in marked variability in predicting later onset of PTSD symptoms at 1 and 4 months after injury. It is possible that this variability may be attributed to the design of the SASRQ, the study retention and participation rate, or the opt-in as opposed to opt-out study design. The variability may also be due to a transient stress response that remitted within the first month after injury given the reduction in sensitivity, specificity, and power to predict PTSD between months 1 and 4. Although these finding suggest that the ASD diagnosis or its symptoms are not the ideal clinical detection tools for predicting later onset of PTSD or other stressors, the initial evidence suggests it is more efficient than current practice.

The sensitivity, specificity, and power to predict PTSD using the SASRQ questionnaire did produce rates that conform with the current literature. In a recent system review of thirteen adult injury-related acute stress studies, the ASD diagnosis resulted in a mean sensitivity of $0.50(0.29-0.89)$, specificity of $0.89(0.56-0.97)$, and power to predict PTSD of $0.54(0.25-0.82)$ using various questionnaires over periods ranging from 2 to 
Table 4 Characteristics of participants by response scores to the PCL-S PTSD self-assessment test at month 1 and month 4

\begin{tabular}{|c|c|c|c|c|c|c|}
\hline Variable & $\begin{array}{c}\text { Symptom }+(n=14) \\
\text { month } 1\end{array}$ & $\begin{array}{l}\text { Symptom - }(n=42) \\
\text { month } 1\end{array}$ & $\begin{array}{c}p \\
\text { value }\end{array}$ & $\begin{array}{l}\text { Symptom }+(n=12) \\
\text { month } 4\end{array}$ & $\begin{array}{c}\text { Symptom }-(n=31) \\
\text { month } 4\end{array}$ & $p$ value \\
\hline \multicolumn{7}{|l|}{ Socio-demographic factors } \\
\hline Mean (SD) age (years) & $45(20)$ & $46(19)$ & 0.81 & $45(15)$ & $49(20)$ & 0.50 \\
\hline Male & $6(42)$ & $33(78)$ & 0.03 & $8(67)$ & $22(71)$ & 1.00 \\
\hline Having no high school diploma & $3(21)$ & $3(7)$ & 0.32 & $2(17)$ & $3(10)$ & 0.91 \\
\hline Unemployed at time of injury & $2(14)$ & $6(14)$ & 1.00 & $2(17)$ & $4(13)$ & 1.00 \\
\hline Currently living alone & $3(21)$ & $10(24)$ & 1.00 & $1(8)$ & $5(16)$ & 0.86 \\
\hline Living in rented housing & $10(71)$ & $24(57)$ & 0.53 & $6(50)$ & $18(58)$ & 0.89 \\
\hline \multicolumn{7}{|l|}{ Health status factors } \\
\hline $\begin{array}{l}\text { Treated for anxiety or depression in the } \\
\text { last year }\end{array}$ & $3(21)$ & $8(19)$ & 1.00 & $4(33)$ & $3(10)$ & 0.17 \\
\hline Has consulted with GP/care provider & $6(54)$ & $11(38)$ & 0.55 & $10(91)$ & $11(42)$ & 0.02 \\
\hline Currently taking medication for stress & $7(50)$ & $15(36)$ & 0.53 & $5(42)$ & $3(10)$ & 0.05 \\
\hline Feel as healthy as prior to injury & $3(21)$ & $8(19)$ & 1.00 & $4(33)$ & $3(10)$ & 0.17 \\
\hline $\begin{array}{l}\text { Had not expected pain to still be } \\
\text { present }\end{array}$ & $12(85)$ & $33(78)$ & 0.85 & $9(75)$ & $27(87)$ & 0.61 \\
\hline Has experienced barriers obtaining care & $1(7)$ & $2(5)$ & 1.00 & $5(42)$ & $2(6)$ & 0.02 \\
\hline \multicolumn{7}{|l|}{ Injury-related factors } \\
\hline $\begin{array}{l}\text { Positive blood alcohol (BAC) upon } \\
\text { admission }\end{array}$ & $1(8)$ & $6(16)$ & 0.86 & $2(18)$ & $3(11)$ & 0.92 \\
\hline $\begin{array}{l}\text { Positive toxicology result upon } \\
\text { admission }\end{array}$ & $1(8)$ & $5(13)$ & 1.00 & $1(9)$ & $2(7)$ & 1.00 \\
\hline Mean (SD) Injury severity score (ISS) & $15(14)$ & $24(12)$ & 0.03 & $18(14)$ & $24(12)$ & 0.24 \\
\hline Cause of injury was intentional & $2(17)$ & $2(5)$ & 0.48 & $2(17)$ & $1(3)$ & 0.41 \\
\hline
\end{tabular}

Values reported are counts (\% of group total) unless otherwise stated.

24 months after injury [9]. In comparison to ad hoc clinical referral practices, routine screening improved the identification of patients whose acute stress persisted within the first month after injury. Although ad hoc referral resulted in greater predictive power after 4 months, this improvement came at the expense of identifying fewer individuals with acute stress. When contrasted against the context of patient experiences, these preliminary results suggest routine as opposed to ad hoc screening for acute stress is a more sensitive approach for identifying populations who would benefit from interventions.

Our estimation of acute stress under current practice may be conservative due to the reliance on patient discharge summary notes to identify in-hospital referrals with psychiatric services. As the level of accuracy in the number of psychiatric referrals that are recorded in patient discharge summaries is unknown, this study does provide a reference point from which to gauge future assessments. However, it may be that current practices over estimate incidence of acute stress as all persons with a previous mental health history are automatically identified in the patient registry and flagged for a consult after admission to Trauma Services. In this study, five of the eight individuals who were consulted by psychiatry prior to discharge met this criterion.

Research has shown an estimated 13 to $25 \%$ of unintentional and intentional injury survivors display symptoms of acute stress following injury [23-25]. The prevalence of acute stress identified in this study from routine screening confirms with the current literature whereas the ad hoc referral underestimates the frequency that patients experience this type of stress response. However, using the ASD diagnosis as a means to reduce the risk of PTSD remains a challenge due to limitations of screening, the lack of capacity to provide care, and the inconclusive evidence on the effectiveness of treatment [11]. In addition, it has been shown that therapies to minimize PTSD other than cognitive behavior therapy, such as behavioral activation, cognitive restructuring, counseling, relaxation therapy, stepped collaborative care, or structured writing interventions are largely ineffective [26]. While there remains a need to abstain from providing a clinical diagnosis of PTSD in absence of capacity to provide care, this reservation is not sufficient grounds to avoid routine screening for acute stress as it would detract from the capacity to understand broader contexts associated with recovery and how best to 
anticipate and respond to patient needs prior to and after discharge.

Our preliminary findings suggest that the definitive study should test whether an improved discharge management program improves patient outcomes. Our initial results suggest that patients lack knowledge of resources available during recovery, are dissatisfied with their level of social support to help them manage their health, under-utilizing health care services as a result of either insufficient knowledge or financial resources, and that psychological stressors are largely unexpected. Some of these experiences could be prevented through improving discharge practices. For example, in British Columbia all individuals are eligible to apply for temporary premium health care assistance to reduce the financial impact of obtaining extended health care (e.g. physiotherapy, counseling) in the event that they do not qualify for premium assistance or would exhaust their health insurance to maintain preventative health care treatment. Only one participant was knowledgeable of this insurance assistance program, suggesting that greater patient advocacy can be incorporated into routine practice. In addition, previous studies have initiated supportive self-management programs to increase the effectiveness of treatment for depression [27]. A supportive self-management program targeted at survivors who exhibit acute or longterm symptoms of stress could be tested to determine whether post-discharge care management that emphasizes coaching and behavior change can foster selfefficacy with respect to their emotional health.

We developed a systematic enrollment program to identify participants for follow-up based on daily coordination between the trauma nurse coordinator and the research team. Following rounds, the trauma nurse coordinator provided a list of all patients to be discharged in the afternoon or who were likely to be discharged on the following day. There were few occasions where more than two individuals were discharged on a single day, thereby making it feasible that one individual could conduct the initial enrollment interview. An underlining benefit of this approach is that the coordinator serves as a focal point for daily trauma operations and is in a strong position to broker additional support from clinical staff and surgical residents as to the benefits of improved patient follow-up. Limitations of this approach were that persons discharged on weekends were missed. For the definitive study, enlisting surgical residents to coordinate baseline interviews during weekends could help minimize this source of potential bias. A retrospective review of trauma registry records found no difference between consenters and patients who were missed with respect to age, sex, injury intent, BAC, or toxicology result.

Importantly, this pilot study is hindered by the limitation of a sample of 91 individuals. This limitation prohibited a more robust analysis of covariates attributed to retention and psychological morbidity, both of which could help identify whether targeted as opposed to universal screening would be more beneficial. While participants who maintained follow-up throughout the entire study period had similar health and socio-economic backgrounds, there were differences among those who withdrew from follow-up from those who maintained full or partial participation. These differences may lead to participation bias and strategies to minimize their impact will need to be addressed. It should be noted that the study was completed prior DSM-V and used a survey that was developed using the DSM-IV criteria.

\section{Conclusion}

This pilot study suggests there are opportunities to enhance current discharge practices in trauma care by actively screening for ASD symptomatology. This would represent a practice change in trauma care, but it is no means certain other trauma centers are able or willing to take on additional screening. To our knowledge, this shift in care has not yet been undertaken elsewhere in Canadian trauma centers. Thus far, representatives of our Quality Council have encouraged continued screening for acute stress and have supported its inclusion as part of standard care practice. As such, we have proposed a quaternary discharge survey whereby acute stress assessments would become part of routine patient handover prior to discharge. The proposal was framed such that future surveys would be undertaken by our surgical residents, under the rationale that this practice emphasizes the 'Health Advocacy' component of the Royal College of Physicians and Surgeons of Canada CanMEDS Physician Competency Framework - traditionally one of the least supported CanMEDS roles. Further research into the epidemiology of acute stress and assessment of patient outcomes is required to assess whether this is an optimal structural framework for minimizing psychological morbidity after injury.

\section{Competing interests}

There are no competing interests among any of the authors.

\section{Authors' contributions}

Conception and design: NB and RKS. Acquisition of data: NB. Analysis and interpretation of data: NB, RKS, BS, SA, RH. Drafting of manuscript: NB, RKS, BS, SA. Study supervision: RKS and BS. All authors read and approved the final manuscript.

\section{Acknowledgements}

The authors would like to thank Tracey Taulu, Kara George, Lori Quinn, Lois Budd, Court Babcock, and Margret Little from Vancouver General Hospital.

The authors would also like to acknowledge the support of the BC Trauma Registry and to Drs. Lynne Moore and Dirk Stengel for reviewing this manuscript and their helpful comments and suggestions. Funding for NB was supported by a postdoctoral fellowship awarded from the Canadian Institutes of Health Research (CIHR). 


\section{Author details}

${ }^{1}$ Department of Surgery, University of British Columbia, 855 West 10th Avenue, Vancouver, British Columbia V5Z 1 M9, Canada. ${ }^{2}$ School of Population and Public Health, University of British Columbia, 2206 East Mall, Vancouver, British Columbia V6T 1Z3, Canada. ${ }^{3}$ Department of Psychiatry, University of British Columbia, 855 West 10th Avenue, Vancouver, British Columbia V5Z 1 M9, Canada. ${ }^{4}$ College of Nursing, University of South Carolina, 1601 Greene Street, Columbia, SC 29208, USA

Received: 15 November 2012 Accepted: 29 April 2014

Published: 5 May 2014

\section{References}

1. Bryant RA, Creamer M, O'Donnell ML, Silove D, McFarlane AC: A multisite study of the capacity of acute stress disorder diagnosis to predict posttraumatic stress disorder. J Clin Psychiatry 2008, 69:923-929.

2. Shalev AY, Tuvia P, Canetti L, Schreiber S: Predictors of PTSD in injury trauma survivors: a prospective study. Am J Psychiatry 1996, 153:219-225.

3. Shalev AY, Freedman S, Peri T, Brandes D, Sahar T, Orr SP, Pitman RK. Prospective study of posttraumatic stress disorder and depression following trauma. Am J Psychiat 1998, 155(5):630-637.

4. Michaels AJ, Michaels CE, Moon CH, Smith JS, Zimmerman MA, Taheri PA, Peterson C: Posttraumatic stress disorder after injury: impact on general health outcome and early risk assessment. J Trauma 1999, 47:460-467.

5. McKibben JB, Bresnick MG, Wiechman SA, FJ A: Acute stress disorder and posttraumatic stress disorder: a prospective study of prevalence, course, and predictors in a sample with major burn injuries. J Burn Care Res 2008, 29:22-35.

6. Harris IA, Young JM, Rae H, Jalaludin BB, Solomon MJ: Predictors of posttraumatic stress disorder following major trauma. ANZ J Surg 2008, 78(7):583-587.

7. Zlotnick C, Rodriguez BF, Weisberg RB, Bruce SE, Spencer MA, Culpepper L, Keller MB: Chronicity in posttraumatic stress disorder and predictors of the course of posttraumatic stress disorder among primary care patients. J Nerv Ment Dis 2004, 192(2):153-159.

8. Benitez CIP, Zlotnick C, Stout RI, Lou FJ, Dyck I, Weisberg R, Keller M: A 5-year longitudinal study of posttraumatic stress disorder in primary care patients. Psychopathology 2012, 45(5):286-293

9. Bryant RA: Acute stress disorder as a predictor of posttraumatic stress disorder: a systematic review. J Clin Psychiatry 2011, 72(2):233-239.

10. Zatzick D, Jurkovich GJ, Rivara FP, Wang J, Fan MY, Joesch J, Mackenzie E: A national US study of posttraumatic stress disorder, depression, and work and functional outcomes after hospitalization for traumatic injury. Ann Surg 2008, 248(3):429-435.

11. Shalev AY, Ankri YLE, Israeli-Shalev Y, Peleg T, Adessky R, Freedman S: Prevention of posttraumatic stress disorder by early treatment: results from the Jerusalem trauma outreach and prevention study. Arch Gen Psychiatry 2012, 69:166-176.

12. Adler D, Bungay K, Wilson I, Pei Y, Supran S, Peckham E, Cynn DJ, Rogers $\mathrm{WH}$ : The impact of a pharmacist intervention on 6-month outcomes in depressed primary care patients. Gen Hosp Psychiatry 2004, 26(3):199-209.

13. Jaycox L, Miranda J, Meredith L, Duan N, Benjamin B, Wells K: Impact of a primary care quality improvement intervention on use of psychotherapy for depression. Ment Health Serv Res 2003, 5(2):109-120.

14. Schoenbaum M, Unutzer J, McCaffrey D, Duan N, Sherbourne C, Wells KB: The effects of primary care depression treatment on patients' clinical status and employment. Health Serv Res 2002, 37(5):1145-1158.

15. Edens E, Kasprow W, Tsai J, Rosenheck R: Association of substance use and VA service-connected disability benefits with risk of homelessness among veterans. Am J Addict 2011, 20:412-419.

16. Van Beeck EF, Larsen CF, Lyons RA, Meerding W-J, Mulder S, Essink-Bot M-L: Guidelines for the conduction of follow-up studies measuring injuryrelated disability. J Trauma-Inj Infect Crit Care 2007, 62(2):534-550.

17. Cardena E, Koopman C, Classen C, Waelde LC, Spiegel D: Psychometric properties of the stanford acute stress reaction questionnaire (SASRQ): a valid and reliable measure of acute stress. J Trauma Stress 2000, 13:719-734

18. Classen C, Koopman C, Hales R, Spiegel D: Acute stress disorder as a predictor of posttraumatic stress symptoms. Am J Psychiatry 1998, 155:620-624.
19. Koopman C, Gore-Felton C, Classen C, Kim P, Spiegel D: Acute stress reactions to everyday stressful life events among sexual abuse survivors with PTSD. J Child Sex Abus 2001, 10:83-99.

20. Weathers F, Litz B, Herman D, Huska J, Keane T, The PTSD Checklist (PCL): Reliability, Validity, and Diagnostic Utility. San Antonio TX: Paper presented at the annual meeting of the International Society for Traumatic Stress Studies: 1993

21. Blanchard EB, Jones-Alexander J, Buckley TC, Forneris CA: Psychometric properties of the PTSD checklist (PCL). Behav Res Ther 1996, 34:669-673.

22. SAS Institute Inc: SAS/STAT ${ }^{\oplus}$ Version 9.2 of the SAS System for Windows. Cary, NC, USA: Copyright (c) 2008 SAS Institute Inc; 2009.

23. Brewin CR, Andrews B, Rose S, Kirk M: Acute stress disorder and posttraumatic stress disorder in victims of violent crime. Am J Psychiatry 1999, 156:360-366.

24. Holeva V, Tarrier N, Wells A: Prevalence and predictors of acute stress disorder and PTSD following road traffic accidents: thought control strategies and social support. Behav Ther 2001, 32:65-83.

25. Elklit A: Acute stress disorder in victims of robbery and victims of assault. J Interpers Violence 2002, 17:872-887.

26. Bisson J, Andrew M: Psychological treatment of post-traumatic stress disorder (PTSD). Cochrane Database Syst Rev 2007, 3: Art. No.: CD003388.

27. Katon W, Unutzer J, Simon G: Treatment of depression in primary care where we are, where we can go. Med Care 2004, 42:1153-1157.

doi:10.1186/1752-2897-8-5

Cite this article as: Bell et al.: Routine versus ad hoc screening for acute stress following injury: who would benefit and what are the opportunities for prevention. Journal of Trauma Management \& Outcomes 2014 8:5.

\section{Submit your next manuscript to BioMed Central and take full advantage of:}

- Convenient online submission

- Thorough peer review

- No space constraints or color figure charges

- Immediate publication on acceptance

- Inclusion in PubMed, CAS, Scopus and Google Scholar

- Research which is freely available for redistribution 